\title{
Experimental shifts in egg-nest contrasts do not alter egg rejection responses in an avian host-parasite system
}

Mark E. Hauber 1,*

Email Mark.Hauber@Hunter.CUNY.edu

Zachary Aidala 1,2

Branislav Igic 3

Matthew D. Shawkey 3

Csaba Moskát 4,5

1 Department of Psychology, Hunter College and the Graduate Center, City University of New York, 695 Park Avenue, New York, NY, 10065 USA

2 Social and Behavioral Sciences Division, Bloomfield College, 467 Franklin Street, Bloomfield, NJ, 07003 USA

3 Department of Biology, University of Akron, Akron, OH, 44325-3908 USA

4 MTA-ELTE-MTM Ecology Research Group, Hungarian Academy of Sciences, c/o Biological Institute, Eötvös Lóránd University, Pázmány Péter sétány 1/C., Budapest, 1117 Hungary

5 Hungary and Hungarian Natural History Museum, Baross u.

13., Budapest, 1088 Hungary

\section{Abstract}


Obligate brood parasitic birds exploit their hosts to provide care for unrelated young in the nest. Potential hosts can reduce the cost of parasitism by rejecting foreign eggs from the nest. Observational, comparative, and experimental studies have concluded that most hosts use the coloration and patterning of eggshells to discriminate between own and foreign eggs in the nest. However, an alternative hypothesis is that birds use the colour contrasts between eggshells and the nest lining to identify parasitic eggs (egg-nest contrast hypothesis). In support of this hypothesis, we found that the avian perceivable chromatic contrasts between dyed eggs and unmanipulated nest linings significantly and negatively covaried with the rejection rates of different dyed eggs of the great reed warbler Acrocephalus arundinaceus, a frequently parasitized host of the common cuckoo Cuculus canorus. To experimentally test whether egg-nest contrasts influence rejection, we reciprocally dyed both eggs and the nest lining of this host species with one of two colours: orange and green. Contrary to the egg-nest contrast hypothesis, host rejection patterns in response to dyed eggs were not altered by dyeing nests, relative to unmanipulated control eggs and nests. In turn, experimental egg colour was the only significant predictor of egg rejection rate. Our results demonstrate that egg-nest contrast is a collateral, not a causal factor in egg rejection, and confirm the conclusions of previous studies that hosts can rely on the parasitic egg's appearance itself to recognize the foreign egg in the nest.

\section{AQ1 \\ $\mathrm{AQ2}$ \\ AQ3}

\section{Keywords}

Chromatic contrast

Coevolution

Egg rejection

Perceptual modelling

\section{Introduction}


Brood parasitic birds reduce the reproductive success of their hosts by imposing costly parental care for unrelated progeny (Davies 2000, 2011). Hosts may lower or eliminate the recoverable costs of parasitism (Croston and Hauber 2015a) by ejecting foreign eggs (Kilner and Langmore 2011) or deserting parasitized nests (Hauber et al. 2014). Numerous studies have demonstrated that egg rejection can be predictably caused by manipulating several traits of parasitic eggs, including their size (Stokke et al. 2010; Guigueno et al. 2014), shape (Zölei et al. 2012), coloration (Honza et al. 2007), and/or maculation pattern (Moskát et al. 2008). Overall, host species are more likely to reject parasitic eggs when there are greater differences in appearance between the parasite's and the host's own eggs (Rothstein 1982; Samas et al. 2011; de la Colina et al. 2012; Soler et al. 2012; Abernathy and Peer 2014).

AQ4

The examination of egg phenotypes from a bird's perspective, using an opponent mechanism-based perceptual modelling approach of avian vision (Vorobyev and Osorio 1998), has further improved our understanding of avian host-parasite coevolution. These studies assess chromatic reflectance and/or pattern data to characterize how the tetrachromatic avian visual system assesses the foreign eggshell's appearance (Avilés et al. 2010; Stoddard and Stevens 2011; Hanley et al. 2013; Igic et al. 2012; Poláček et al. 2013; Stoddard et al. 2014), and have repeatedly confirmed long-standing conclusions that foreign eggs are rejected more often when they are perceived as more dissimilar to the hosts' own eggs (Cassey et al. 2008; Spottiswoode and Stevens 2010; Stevens et al. 2013; Croston and Hauber 2014; Hauber et al. 2015; Fig. 1 ).

\section{Fig. 1}

Three steps in the assessment of the potential role of egg-nest chromatic contrasts in egg rejection decisions by great reed warbler hosts of the common cuckoo. a The relationship between egg rejection rates and egg-egg contrasts plotted as the mean just noticeable differences (JNDs) between unmanipulated host eggs versus dyed host eggs, unmanipulated eggs of the cuckoo, and unmanipulated other conspecifics of the hosts (data replotted from Hauber et al. 2015). b The relationship between egg-nest contrast, plotted as the mean JNDs between unmanipulated host nest lining 
versus dyed host eggs, unmanipulated eggs of the cuckoo, and unmanipulated other conspecifics of the hosts, and the egg-egg contrasts from a. c The relationship between egg rejection rates and egg-nest contrasts from (b)
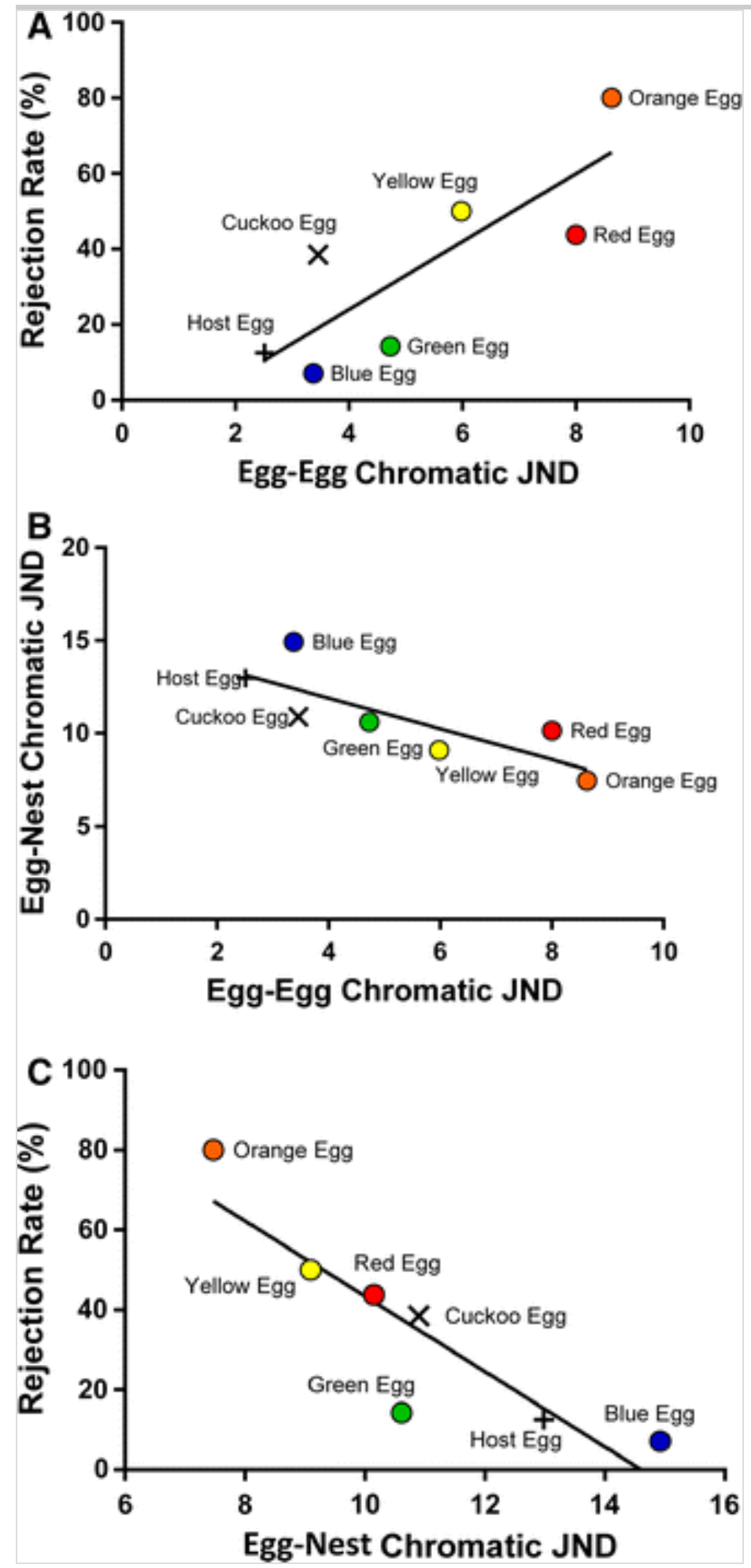
However, egg rejection behaviours of hosts are also influenced by the physical and biotic properties of the nest environment. Some of these factors include the intensity (Langmore et al. 2005) and composition (Honza et al. 2011) of the light illuminating the nest (Honza et al. 2014), the number and colour of the other eggs in the nest (Lang et al. 2014; Moskát et al. 2014a; Yang et al. 2014), variation in the arrangement of the whole clutch (Polaciková et al. 2013; but see Hanley et al. 2015 ), and the prior presence of parasitic eggs in the clutch (Hauber et al. 2006; Moskát and Hauber 2007; Moskát et al. 2014b). Similarly, important factors include the date of clutch initiation by the host (de Mársico et al. 2013), and the presence of brood parasitic adults near the nest or in the breeding habitat (Davies and Brooke 1988; Mosknes and Røskaft 1989; Bártolet al. 2002). Moreover, some brood parasites have evolved eggs that do not mimic the host egg, but instead are cryptic or dark in enclosed host nests with poor illumination (Langmore et al. 2009 ), to evade detection and rejection either by the hosts or by other brood parasites laying in the same host nests (Gloag et al. 2014). The acceptance of dark, low-luminance eggs suggests that specific background matching, or low overall contrast between the eggshell and the nest lining, may also play a role in the (lack of) detection and recognition of foreign eggs in the nests (Mason and Rothstein 1987; Langmore et al. 2005, 2009). Taken together, these studies imply that an egg's immediate milieu can contribute to the rejection of parasitic eggs (Hauber $2014)$.

In nearly all egg rejection studies, to date researchers have conducted experiments by replacing/adding a model/real egg with a different colour/pattern from the host's own eggs (Davies 2000; Hauber et al. 2015) into an active host nest. However, there are alternative explanations for the observed egg rejection patterns in these studies. For example, it may not be that the foreign egg's colour per se causes rejection, but rather it is caused the perceived visual contrast between the egg's appearance and that of the materials lining the nest (e.g. Igic et al. 2009). Accordingly, egg-nest contrast could directly influence foreign egg recognition and rejection (Moskát and Hauber 2007; Antonov et al. 2009). If so, then hosts may easily detect and reject all objects, including parasitic eggs, detritus, broken eggs leaking yolk, or hatched eggs with bright white interiors (Tinbergen et al. 
1962; Guigueno and Sealy 2012) when they exceed a threshold contrast typical between own eggs and nest lining.

To date, only one study has directly assessed whether avian perceivable egg-nest contrast per se is a proximate cause of foreign egg rejection by hosts of brood parasitic birds: Aidala et al. (2015) found no causal relationship between egg-nest contrast and the rejection of red, blue (host mimetic), and beige (parasite mimetic) plaster eggs by American robins Turdus migratorius, an egg rejecter host of the generalist and non-mimetic brood parasitic brown-headed cowbirds Molothrus ater in North America (Croston and Hauber 2015b). Parallel studies reported here have focused on the great reed warbler Acrocephalus arundinaceus, a commonly parasitized host of a highly mimetic host race of the common cuckoo Cuculus canorus in Hungary (Moskár) and elsewhere (Drobniak et al. 2014). In this species, egg-egg avian perceivable chromatic contrast between parasitic/foreign eggs and the hosts' own eggs positively predicts egg rejection rate across a range of natural and artificial colours (Hauber et al. 2015; Fig. 1). However, as also seen in the American robin (Aidala et al. 2015), egg-egg contrast is negatively correlated with egg-nest contrasts between experimental eggs and the natural nest lining's coloration (Fig. 1). This statistical relationship makes egg-nest contrast a significant, albeit negative predictor of egg rejection rates (Fig. 1). This is contrary to the egg crypsis hypothesis for the acceptance of dark foreign eggs (Gloag et al. 2014), but still implies a potentially causal relationship between eggnest contrast and egg rejection patterns in great reed warblers.

Thus, egg colour manipulation experiments in this host can be confounded by collateral changes in egg-nest contrasts, requiring a direct experimental assessment of the relative role of egg-egg versus egg-nest contrast in egg rejection by great reed warblers. To fill this gap in our knowledge, we collated published data and conducted new experiments in which we reciprocally and independently manipulated the appearance of both the eggs and the nest lining by dyeing them one of two different colours (Fig. 2). We predicted (based on Fig. 1) that egg colours with experimentally lower contrasts against the dyed nest are more likely to be rejected.

\section{Fig. 2}


Appearance and reflectance properties of the experimental egg and nest stimuli used in this study. a Photographic representations and representative reflectance spectra of the experimental treatment of dyeing eggs and nest linings. b Avian perceivable chromatic contrasts between natural versus natural or dyed eggs (mean $\pm \mathrm{SE}$ shown) vary significantly $\left(F_{3,24}=19.2, P<0.0001\right)$. Bars with different letters inside are significantly different as per post hoc student tests, with $P$ values adjusted for multiple comparisons (Holm 1979). The pairwise comparisons refer to specific stimulus types: $N_{e}$ natural (unmanipulated) host egg, $O_{e}$ orange-dyed egg, and $G_{e}$ green-dyed egg. c Avian perceivable chromatic contrasts (JNDs) between natural or dyed eggs and natural or dyed nest linings (mean \pm SE shown). Relevant statistical comparisons are shown in the main text. The pairwise comparisons refer to specific stimulus types: $N_{e}$ natural (unmanipulated) host egg, $O_{e}$ orange-dyed egg, $G_{e}$ greendyed egg, $N_{n}$ natural (unmanipulated) nest, $O_{n}$ orange-dyed nest, and $G_{n}$ green-dyed nest 

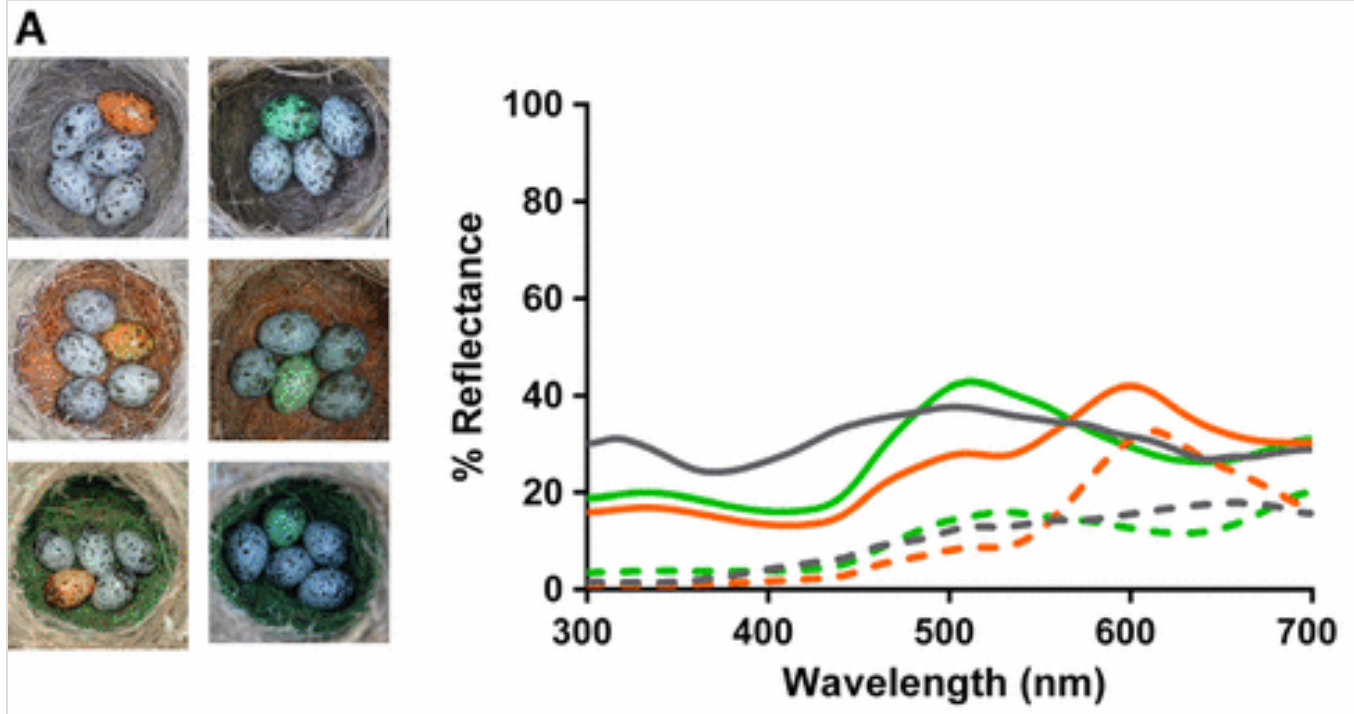

- Natural Egg

- - Natural Nest

- Orange Eggs

- - Orange Nest

- Green Eggs

- - Green Nest
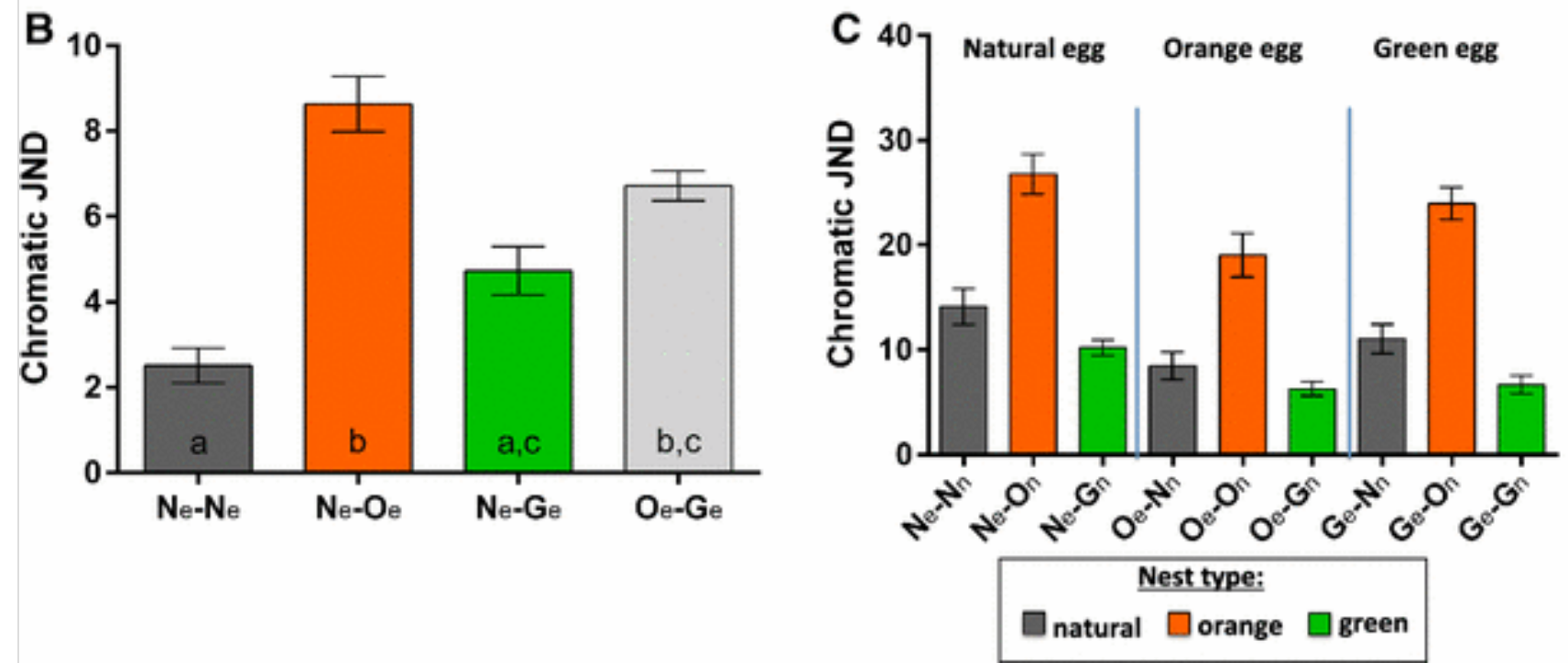

\section{Materials and methods}

\section{Experimental egg rejection data from natural nests}

We studied egg ejection behaviours of great reed warblers $A$. arundinaceus, a frequently parasitized host of the common cuckoo C. canorus, in the surroundings of Apaj $\left(47^{\circ} 07^{\prime} \mathrm{N} ; 19^{\circ} 06^{\prime} \mathrm{E}\right)$, Central Hungary. We compiled our own published results on egg rejection responses of this host species (Bán et al. 2013); these data are comprised of the percentage of nests at which one of the host's own eggs was dyed one of several colours and were subsequently rejected. To manipulate eggshell appearance in this study, we used a long-lasting, non-toxic, soft-tip highlighter pen (Stabilo Boss ${ }^{\mathrm{TM}}$ ) to cover the egg surface with one of five artificial 
colours (blue, green, orange, red, and yellow, $N=12-16$ eggs per colour; for spectral details, methodology, and egg rejection results, see Bán et al. 2013 ). We collated additional data on the experimental rejection rates of a single unmanipulated conspecific ( $N=16$, Bártol et al. 2002) or a common cuckoo egg $(N=13$; Hauber et al. 2015) inserted into active, non-parasitized great reed warbler nests at the same study site during prior years; for all of these data sources, we used the percentage of eggs rejected per stimulus type within the 6-day monitoring period.

\section{Egg rejection experiments in dyed nests}

To experimentally study the role of chromatic contrast between eggs and nests, we selected two of the five artificial egg colours, used in previous studies with natural nest linings, that had yielded disparate rejection rates (orange egg: high rejection rate at $77 \%$; green egg: low rejection rate at $14 \%$ ) by great reed warblers (Bán et al. 2013 ; Fig. 3 ). During May-June 2013 and 2014, we used orange (Stabilo Boss $70 / 54$, Fig. 1 a) or green (Stabilo Boss 70/33) highlighter pens to dye one of the hosts' own eggs in the nest.

\section{Fig. 3}

Egg rejection rates of dyed eggs in natural or dyed nests. The rejection rates refer to the following egg types in the following nest types: $N_{e}$ natural (unmanipulated) host egg, $O_{e}$ orange-dyed egg, $G_{e}$ green-dyed egg, $N_{n}$ natural (unmanipulated) nest, $O_{n}$ orange-dyed nest, and $G_{n}$ green-dyed nest 


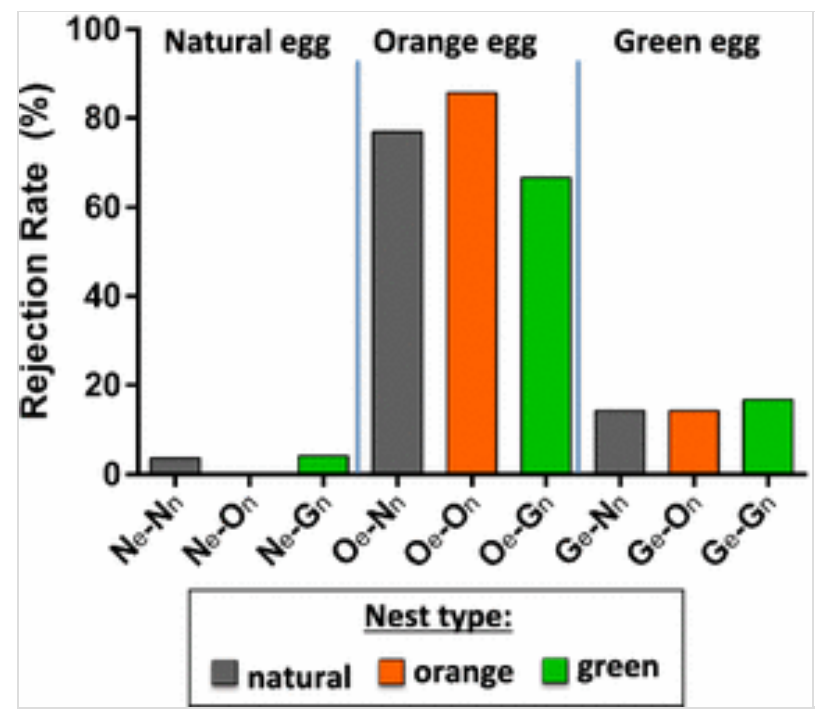

To independently manipulate nest colour and, thus, to shift the relative contrast between eggs and nests, we also dyed the nest lining orange (2013), or green (2014), in experimental nests. Nests were dyed by running the soft tip of pens thoroughly over the grass stalks and other nest lining materials to generate full coverage (Fig. 1). Once the solvent of the highlighter pen dried, the dye remained set and did not bleed onto other eggs in the nest. As we used highlighter pens for dyeing the experimental eggs and nests following previous works on experimental parasitism (e.g. Bán et al. 2013; Moskát et al. 2014a, b), the resulting colours were the combination of the original background and the highlighter's dye (for the representative spectra, see Fig. 1). For experimental controls and comparisons, we used the following data: egg rejection rates of natural eggs in natural nests (from Bán et al. 2013), of natural eggs in orange or green nests (this study), and of orange and green eggs in natural nests (also from Bán et al. 2013).

\section{AQ6}

Altogether, our manipulations generated new data for $N=14$ orange and $N=14$ green eggs in $N=28$ orange-dyed nests and $N=12$ orange and $N=12$ green eggs in $N=24$ green-dyed nests. To parallel our experimental parasitism methods already published for the non-manipulated, natural nests (hereafter: natural nests; $N=13$ single orange eggs and $N=14$ single green eggs in $N=27$ nests, from Bán et al. 2013 ), our new nest lining and egg colour manipulations also took place when the host clutch was completed (5 eggs, rarely 4 or 6 ). The nest content was then monitored for a standard 6 consecutive days (Bán et al. 2013). Experimental 
eggs that remained in the nest for 6 days were deemed accepted, and eggs that went missing from the nest between subsequent daily visits were considered ejected (sensu Bán et al. 2013). Nest desertion is not a response to experimental parasitism using a single dyed host egg per nest in this host species (Hauber et al. 2006), and so ejection and rejection are used interchangeably throughout this report.

Only active host nests without natural cuckoo parasitism, and unaffected by predation or desertion, were included in this study. Dyeing the nest lining orange or green did not negatively affect the hosts' nesting behaviours: no nests were abandoned as a result of the manipulation and only in one case did nonmanipulated (natural) eggs go missing ( 2 host eggs, as well as an orange egg in a green-dyed nest), right after a thunderstorm.

All work was approved by the local authorities to follow guidelines for the ethical use of wild animals in research. We acknowledge that our natural and experimental nest treatments were conducted in different years; however, annual differences in the rejection rates of experimentally dyed eggs are not a known confounding factor in this host species (Moskát et al. 2009), despite some annual variation in the great reed warbler's egg appearance (Honza et al. 2012) and, likely, also nest appearance. To mediate this concern, we also report and statistically analyse egg rejection rates of the hosts' own eggs from each year and each egg-nest manipulation experiment as an internal control.

\section{Spectral data of eggs and nests}

We collated published reflectance spectra of natural or dyed eggs $(N=10$ /colour $)$ originally measured across the avian visible range $(300-700 \mathrm{~nm})$ with Ocean Optics USB 2000 spectrophotometers (from Igic et al. 2012; Bán et al. 2013). For the current study, we also collected new reflectance data at four spots covering each of the quadrants of the bottom portion of materials lining the nest, where the eggs make direct contact with the nest, for both natural $(N=6)$ and dyed $(N=6$ orange and $N=6$ green) nests. We used averaged reflectance data per egg or nest, from a subset of all eggs and nests used in these and prior experiments for our perceptual modelling. However, we did so from different eggs and nests and across 
years; thus, we do not have egg rejection responses for specific eggs and nests with known spectra and egg-nest contrasts. Critically, however, our focal statistical analyses are based not on the egg-nest specific visual contrast values (see below) but, instead, directly on the experimental treatments themselves (dyed egg colour $\times$ dyed nest colour).

\section{Perceptual modelling and analyses of visual contrasts}

To model the avian visual perception of eggs in the nest, we employed methods following the approaches developed by Vorobyev and Osorio (1998) for comparing colours as birds see them. We generated randomized, unique pairs of egg and nest spectra within our data set for which we calculated avian perceivable contrasts as just noticeable differences (JNDs). We implemented perceptual modelling using the package 'pavo' (Maia et al. 2013) in R (R Core Team 2013 ). In the absence of known physiological data for great reed warblers, we used average photoreceptor data for an ultraviolet sensitive (UVS) bird (300-700 nm), forest shade ambient light spectrum, and a ratio of UVS 1:SWS 1.78:MWS 2.21:LWS 1.96 for the density of the four avian photoreceptor classes (Hart et al. 2000 ). We used a Weber fraction of 0.5 for the long cone and calculated receptor noise proportionally to the Weber fraction and independent of signal intensity (neural noise). Pavo calculates JNDs separately for achromatic and chromatic components of colour. Achromatic contrast and egg rejection rates of dyed eggs were not statistically related in any of our global or pairwise comparisons (all $P>0.05$ ), and therefore, we did not analyse achromatic contrasts further for this report.

Regarding the use of orange or green pens to dye eggs versus nests, our specific predictions were to reduce the contrast between eggs and nests when dyed the same colour and to increase the contrast between eggs and nests when dyed different colours. In turn, for the data set of the dyed eggs in natural nests or in the data set of the experimentally manipulated egg-nest contrasts, we predicted a positive association or a causal effect, respectively, between egg-nest contrasts and egg ejection rates. We analysed our specific predictions regarding treatments, egg rejection rates, and JNDs using JMP 8.0, with $\alpha=0.05$. 


\section{Results}

\section{Perceptual modelling of egg-nest contrast and rejection rates of eggs in natural nests}

The avian perceivable chromatic contrasts (JNDs) between five artificial egg colours and natural nests showed a trends of a negative relationship with egg-egg contrasts $\left(F_{1,3}=6.8, P=0.08\right)$ but were a negative predictor of the rejection rates of experimentally dyed eggs $\left(F_{1,3}=9.7, P=0.05\right)$ (Fig. 1$)$. The negative relationship between egg-nest contrast and egg-egg contrast became significant $\left(F_{1,5}=8.9, P=0.03\right)$ and between egg rejection rates and egg-nest contrast remained statistically significant $\left(F_{1,5}=19.2, P=0.007\right)$ when following the recommendation of Hauber et al. (2015), and we also included data points for the experimental rejection rates of natural conspecific and cuckoo eggs added to great reed warbler nests (Fig. 1).

\section{Perceptual modelling of experimentally altered contrasts of dyed eggs and nests}

Dyeing the nests orange significantly increased the egg-nest contrast (JNDs) for the host's own eggs from $11.0 \pm 1.4$ (mean \pm SE) in natural nest to $20.1 \pm 2.5$ $\left(t_{10}=3.2, P=0.01\right)$ in orange nests and remained similar between natural and green nests $\left(8.5 \pm 0.7 ; t_{10}=1.6, P=0.14\right)$.

Contrary to the initials aims of our manipulations, dyeing nests orange (Fig. 2) raised the chromatic contrasts of not only the green-dyed eggs but also the orangedyed eggs against the nest lining, relative to the contrast of either egg colour against natural nests (ANOVA, orange dyeing treatment: $F_{2,15}=21.20$, $P<0.0001$; Fig. 2). In turn, as predicted, dyeing the nests green reduced the contrasts with green eggs, but not with orange eggs (green dyeing treatment: $F_{2,15}=48.05, P<0.0001$; Fig. 2 ).

\section{Egg rejection rates in natural and dyed nests}

Regarding the experimental controls, the host's own unmanipulated eggs were accepted in all but two of the 79 nests included in this study (one natural egg was 
ejected from a natural nest and one from a green-dyed nest). Accordingly, there was no statistical effect of the experimental manipulation of nest lining across natural (control) versus dyed (orange and green nests combined) treatments on the rejection rates of the hosts' own eggs (two-tailed Fisher's exact test: $P=0.43$ ).

Overall, the orange-dyed eggs were rejected more often than the green-dyed eggs, irrespective of nest lining colour treatments or the natural, control nests (Fig. 3); statistically, each year/nest treatment within itself yielded a significantly higher rejection rate of orange versus green eggs (all two-tailed Fisher's exact tests: $P<0.05$ : Fig. $1 \mathrm{~d}$ ). When combining all years and treatments together, our full logistic regression model (with predictors: egg treatment, nest treatment, and egg $\times$ nest interaction) significantly predicted egg rejection rates (whole model test $\left.\chi_{5}^{2}=34.31, R^{2}=0.32, P<0.0001\right)$. However, the interaction term between experimental egg colours and nest lining colours was not significant $\left(_{2}{ }^{2}=0.77\right.$, $P=0.68$; Fig. $1 \mathrm{~d}$ ). Thus, we reran the model without an interaction term. Again, only egg colour $\left(\chi_{3}{ }^{2}=33.54, P<0.0001\right)$, but not nest lining colour $\left(\chi_{2}{ }^{2}=0.59\right.$, $P=0.74)$, was a significant predictor of egg rejection in this reduced model.

\section{Discussion}

Great reed warblers in Hungary are parasitized by a host race, or gens, of the common cuckoo that lays the most accurately mimetic eggs of any common cuckoo host race (Moskát et al. 2012). Prior perceptual modelling has revealed that the consistently low chromatic contrasts between the background colours of host and parasite eggs render most cuckoo eggs visually indistinguishable from the host's own eggs (Stoddard and Stevens 2011; Igic et al. 2012; Hauber et al. 2015 ). Yet, these hosts still reject about one-third of naturally laid parasite eggs in the Hungarian population (Moskát et al. 2009), likely by identifying poorly mimetic foreign eggs through both maculation (Moskát et al. 2010) and coloration (Bán et al. 2013 ). Here, we assessed experimentally whether the chromatic contrast between eggs and nests provided a direct, or additional, cue in the detection and rejection parasitic eggs by great reed warblers.

Variation in the avian perceivable chromatic contrasts between experimental egg colours and natural nest linings may explain the statistical pattern of rejection rates 
between differently dyed eggs and egg-nest contrast by great reed warblers (Fig. 1). However, this relationship was in the opposite direction to that predicted by the reduced contrast and crypsis hypothesis. Specifically, the greater the eggnest contrast, the lower the rejection rate (Fig. 1). This relationship remained statistically significant when we included the data points for the rejection rates of unmanipulated conspecific and cuckoo eggs by great reed warblers. Based on these analyses, it may be that hosts use higher chromatic contrasts with the nest lining to detect and not reject own eggs, as was observed in the egg rejection behaviours of the domed-nest-building red bishop Euplectes orix (Honza et al. 2014). A corollary of this new hypothesis is that objects, including parasitic eggs, which are less contrasting than typical host eggs in the nest, may be more likely to be inspected and, eventually, more often rejected by discriminating hosts.

Does chromatic contrast between eggs and nests then serve as a direct cue for egg rejection (whether through increased or decreased contrast), or is it simply an epiphenomenon of differently dyed eggs also having intrinsically different contrasts against the natural nest lining? The results of our focal experimental manipulations of the nest colour, and the resulting egg-nest contrasts, contradict this egg-nest contrast hypothesis: altering nest lining colours did not affect rejection patterns of orange- versus green-dyed eggs. Similarly, additional perceptual modelling calculations showed that, even though dyeing the nests orange or green increased the egg-nest chromatic contrasts of the hosts' own unmanipulated eggs (Fig. 2), natural host eggs disappeared from only one of the dyed nests in this study.

These results thus do not support the hypothesis that egg-nest contrast affects hosts' egg rejection responses. Instead, they confirm the long-standing paradigm that egg appearance per se plays a primary role in the rejection of foreign eggs by hosts of avian brood parasites (Davies 2000): only the colour of the experimentally dyed eggs (orange or green) predicted egg rejection rates across our comparisons between natural and dyed nests (Hauber et al. 2015). The conclusions from our study here are likely robust because the hosts' rejection rates were statistically similar with respect to dyed egg colours, even though the data were derived by an admittedly heterogeneous study protocol, including different 
components of the study conducted with different nest treatments in different years. Nonetheless, our new data are consistent with previous work demonstrating how colour and pattern contrasts between the hosts' own eggs and parasitic eggs positively predict foreign egg rejection rates across diverse host-parasite systems (Cassey et al. 2008; Avilés et al. 2010; Spottiswoode and Stevens 2010; Stevens et al. 2013; Croston and Hauber 2014).

The original intention of our treatments was to reduce the contrast between the orange egg and the orange nest just as we were able to do in the green egg and the green nest treatment (Fig. 2); surprisingly, however, the JNDs of both orange and green eggs were increased by dyeing the nest orange. This may be due to the differential effects of dyeing egg and nests green versus orange: dyeing the nests green yielded a similarly shaped but less bright reflectance spectrum compared to dyeing the eggs green (Fig. 2), likely contributing to low chromatic contrast values (which are not affected by achromatic/brightness differences). In contrast, dyeing the nests orange maintained a sharp reflectance peak at around $600 \mathrm{~nm}$ but reduced reflectance in all other wavelength regions (Fig. 2), resulting in a higher rather than lower perceivable contrast.

Our full experimental design included the reciprocal manipulation of both nests and eggs with two different colours, and we were able to experimentally induce both increased and decreased egg-nest contrasts for green eggs and increased contrast for orange eggs, yet the rejection rates of all these dyed eggs were statistically similar to those seen in natural nests. Therefore, our general conclusion is that the chromatic contrast between eggs and nests per se is not a cause for the pattern of decreased egg rejection of more contrasting eggs in natural nests of great reed warblers. Using eggshell appearance itself, rather than egg-nest contrast, may be beneficial to detect and reject foreign eggs in the nests, because the nest lining itself could be increasingly difficult to focus on and become covered up the increasing number of eggs laid in the nest. Furthermore, even though egg appearances change within days after laying (Moreno et al. 2011), nest appearance might change even more rapidly and more unpredictably, depending on external humidity, the drying up of nesting materials, and nest sanitation and repair behaviours of the parents (Poláček et al. 2013). 
Future experimental work should focus on the potential role of the colour contrast between eggs and nests regarding egg rejection in host species of avian brood parasitic species that lay dark or cryptic eggs in dark and enclosed host nests (Langmore et al. 2009; de Mársico et al. 2013; Gloag et al. 2014). For example, experimenters might consider adding cryptic and dark parasite eggs to nests built or moved to better lit sites, atop more reflective nest lining materials, and/or with experimentally illuminated nest interiors, to assess whether cryptic parasite eggs have evolved in response to birds relying on egg colour luminance itself or relative egg-nest a/chromatic contrasts to recognize foreign eggs in the nest.

\section{Acknowledgments}

These studies were conducted in accordance with local laws and ethical regulations to permit animal research. We are aware of no conflict of interest in this work. For discussions, we thank the referees and the editors, Alexis Brewer Rebecca Croston, Phill Cassey, Ros Gloag, Tomas Grim, Daniel Hanley, Marcel Honza, David Lahti, Naomi Langmore, Peter Samas, Cassie Stoddard, Lainga Tong, and the Shawkey Lab. We are grateful to Nikoletta Geltsch, Miklós Bán, and István Zsoldos for assistance with the experiments in the field. Funding was provided by the Human Frontier Science Program (to MEH and MDS) and by Hungarian National Research Fund (OTKA No. 83217 to CM).

\section{References}

Abernathy VE, Peer BD (2014) Intraclutch variation in egg appearance of Brown-headed Cowbird hosts. Auk 131:467-475

Aidala Z, Croston R, Schwartz J, Tong L, Hauber ME (2015) The role of eggnest contrast in the rejection of brood parasitic eggs. J Exp Biol

$\mathrm{AQ7}$

Antonov A, Stokke BG, Moksnes A, Røskaft E (2009) Evidence for egg discrimination preceding failed rejection attempts in a small cuckoo host. Biol Lett 5:169-171 
Avilés JA, Vikan JR, Fossoy F, Antonov A, Moksnes A, Røskaft E, Stokke BG (2010) Avian colour perception predicts behavioural responses to experimental brood parasitism in chaffinches. J Evol Biol 23:293-301

Bán M, Moskát C, Barta Z, Hauber ME (2013) Simultaneous viewing of own and parasitic eggs is not required for egg rejection by a cuckoo host. Behav Ecol 24:1014-1021

Bártol I, Karcza I, Moskát C, Røskaft E, Kisbenedek T (2002) Responses of great reed warblers Acrocephalus arundinaceus to experimental brood parasitism: the effects of a cuckoo Cuculus canorus dummy and egg mimicry. $\mathrm{J}$ Avian Biol 33:420-425

Cassey P, Honza M, Grim T, Hauber ME (2008) The modelling of avian visual perception predicts behavioural rejection responses to foreign egg colours. Biol Lett 4:515-517

Croston R, Hauber ME (2014) Spectral tuning and perceptual differences do not explain the rejection of brood parasitic eggs by American robins (Turdus migratorius). Behav Ecol Sociobiol 68:351-362

Croston R, Hauber ME (2015a) A recoverable cost of brood parasitism during the nestling stage of the American robin (Turdus migratorius): implications for the evolution of egg rejection behaviors in a host of the Brown-headed cowbird (Molothrus ater). Ethol Ecol Evol 27:42-55

Croston R, Hauber ME (2015b) Experimental shifts in intraclutch egg color variation do not affect egg rejection in a host of a non-egg-mimetic avian brood parasite. PLoS One 10:e121213

Davies NB (2000) Cuckoos, cowbirds and other cheats. T \& AD Poyser, London

Davies NB (2011) Cuckoo adaptations: trickery and tuning. J Zool 284:1-14 
Davies NB, Brooke MdeL (1988) Cuckoos versus reed warblers: adaptations and counteradaptations. Anim Behav 36:262-284

de la Colina MA, Pompilio L, Hauber ME, Reboreda JC, Mahler B (2012) Different recognition cues reveal the decision rules used for egg rejection by hosts of a variably mimetic avian brood parasite. Anim Cogn 15:881-889

de Mársico M, Gloag R, Ursino CA, Reboreda JC (2013) A novel method of rejection of brood parasitic eggs reduces parasitism intensity in a cowbird host. Biol Lett 9:20130076

Drobniak SM, Dyrcz A, Sudyka J, Cichoń M (2014) Continuous variation rather than specialization in the egg phenotypes of cuckoos (Cuculus canorus) parasitizing two sympatric reed warbler species. PLoS One 9:e106650

Gloag R, Keller LA, Langmore NE (2014) Cryptic cuckoo eggs hide from competing cuckoos. Proc R Soc Lond B 281:20141014

Guigueno MF, Sealy SG (2012) Nest sanitation in passerine birds: implications for egg rejection in hosts of brood parasites. J Ornithol 153:35-52

Guigueno MF, Sealy SG, Westphal AM (2014) Rejection of parasitic eggs in passerine hosts: size matters more for a nonejecter. Auk 131:583-594

Hanley D, Stoddard MC, Cassey P, Brennan PLR (2013) Eggshell conspicuousness in ground nesting birds: Do conspicuous eggshells signal nest location to conspecifics? Avian Biol Res 6:147-156

Hanley D, Samaš P, Hauber ME, Grim T (2015) Who moved my eggs? An experimental test of the clutch arrangement hypothesis for the rejection of brood parasitic eggs. Anim Cogn 18:299-305

Hart NS, Partridge JC, Cuthill IC, Bennett ATD (2000) Visual pigments, oil droplets, ocular media and cone photoreceptor distribution in two species of 
passerine bird: the blue tit (Parus caeruleus L.) and the blackbird (Turdus merula L.). J Comp Physiol A 186:375-387

Hauber ME (2014) The book of eggs. University of Chicago Press, Chicago

Hauber ME, Moskát C, Bán M (2006) Experimental shift in hosts' acceptance threshold of inaccurate-mimic brood parasite eggs. Biol Lett 2:177-180

Hauber ME, Samas P, Anderson MG, Rutila J, Low J, Cassey P, Grim T (2014) Life-history theory predicts host behavioural responses to experimental brood parasitism. Ethol Ecol Evol 26:349-364

Hauber ME, Tong L, Bán M, Croston R, Grim T, Waterhouse GIN, Shawkey MD, Barron AB, Moskát C (2015) The value of artificial stimuli in behavioral research: making the case for egg rejection studies in avian brood parasitism. Ethology 121:521-528

Holm S (1979) A simple sequentially rejective multiple test procedure. Scand J Stat 6:65-70

Honza M, Polaciková L, Procházka P (2007) Ultraviolet and green parts of the colour spectrum affect egg rejection in the song trush (Turdus philomelos). Biol J Linn Soc 92:269-276

Honza M, Procházka P, Morongová K, Capek MC, Jelinek V (2011) Do nest light conditions affect rejection of parasitic eggs? A test of the light environment hypothesis. Ethology 117:539-546

Honza M, Procházka P, Pozgayová M (2012) Within- and between-season repeatability of eggshell colouration in the great reed warbler Acrocephalus arundinaceus. J Avian Biol 43:91-96

Honza M, Sulc M, Cherry MI (2014) Does nest luminosity play a role in recognition of parasitic eggs in domed nests? A case study of the red bishop. 
Naturwissenschaften 101:1009-1015

Igic B, Cassey P, Samas P, Grim T, Hauber ME (2009) Cigarette butts form a perceptually cryptic component of Song Thrush Turdus philomelos nests. Notornis 56:134-138

Igic B, Cassey P, Grim T, Greenwood DR, Moskát C, Rutila J, Hauber ME (2012) A shared chemical basis of avian host-parasite egg colour mimicry. Proc R Soc B 279:1068-1076

Lang AK, Bollinger EK, Peer BD (2014) Effect of parasite-to-host egg ratio on egg rejection by a Brown-headed cowbird host. Auk 131:694-701

Langmore NE, Kilner RM, Butchart SHM, Maurer G, Davies NB, Cockburn A, Macgregor NA, Peters A, Magrath MJL, Dowling DK (2005) The evolution of egg rejection by cuckoo hosts in Australia and Europe. Behav Ecol 16:686-692

Langmore N, Stevens M, Maurer G, Kilner RM (2009) Are dark cuckoo eggs cryptic in host nests? Anim Behav 78:461-468

Maia R, Eliason CM, Bitton P-P, Doucet SM, Shawkey MD (2013) Pavo: an R package for the analysis, visualization and organization of spectral data. Methods Ecol Evol 4:906-913

Mason P, Rothstein SI (1987) Crypsis versus mimicry and the color of shiny cowbird eggs. Am Nat 130:161-167

Moreno J, Lobato E, Morales J (2011) Eggshell blue-green colouration fades immediately after oviposition: a cautionary note about measuring natural egg colours. Ornis Fenn 88:51-56

Moskát C, Hauber ME (2007) Conflict between egg recognition and egg rejection decisions in common cuckoo (Cuculus canorus) hosts. Anim Cogn $10: 377-386$ 
Moskát C, Avilés JM, Bán M, Hargitai R, Zölei A (2008) Experimental support for the use of egg uniformity in parasite egg discrimination by cuckoo hosts. Behav Ecol Sociobiol 62:1885-1890

Moskát C, Hauber ME, Avilés JM, Bán M, Hargitai R, Honza M (2009) Increased host tolerance of multiple cuckoo eggs leads to higher fledging success of the brood parasite. Anim Behav 77:1261-1290

Moskát C, Bán M, Székely T, Komdeur J, Lucassen RWG, van Boheemen LA, Hauber ME (2010) Discordancy or template-based recognition? Dissecting the cognitive basis of the rejection of foreign eggs in hosts of avian brood parasites. J Exp Biol 213:1976-1983

Moskát C, Takasu F, Munoz AR, Nakamura H, Bán M, Barta Z (2012) Cuckoo parasitism on two closely-related Acrocephalus warblers in distant areas: a case of parallel coevolution? Chin Birds 3:320-329

Moskát C, Zölei A, Bán M, Elek Z, Tong L, Geltsch N, Hauber ME (2014a) How to spot a stranger's egg? A mimicry-specific discordancy effect in the recognition of parasitic eggs. Ethology 120:616-626

Moskát C, Bán M, Hauber ME (2014b) Naïve hosts of avian brood parasites accept foreign eggs, whereas older hosts fine-tune foreign egg discrimination during laying. Front Zool 11:45

Poláček M, Griggio M, Bartíková M, Hoi H (2013) Nest sanitation as the evolutionary background for egg ejection behaviour and the role of motivation for object removal. PLoS One 8:e78771

Polaciková L, Honza M, Procházka P, Topercer J, Stokke BG (2007) Colour characteristics of the blunt part of blackcap (Sylvia atricapilla) eggs: possible cues for egg recognition. Anim Behav 74:419-427 
Polaciková L, Takasu F, Stokke BG, Moksnes A, Røskaft E, Cassey P, Hauber ME, Grim T (2013) Egg arrangement in avian clutches covaries with the rejection of foreign eggs. Anim Cogn 16:819-828

R Core Team (2013) R: a language and environment for statistical computing. $\mathrm{R}$ Foundation for Statistical Computing, Vienna

Rothstein SI (1982) Mechanisms of avian egg recognition: Which egg parameters elicit responses by rejecter species? Behav Ecol Sociobiol 11:229239

Samas P, Hauber ME, Cassey P, Grim T (2011) Repeatability of foreign egg rejection: testing the assumptions of co-evolutionary theory. Ethology 117:606619

Soler JJ, Avilés JM, Møller AP, Moreno J (2012) Attractive blue-green egg coloration and cuckoo-host coevolution. Biol J Linn Soc 106:154-168

Spottiswoode CN, Stevens M (2010) Visual modeling shows that avian host parents use multiple visual cues in rejecting parasitic eggs. PNAS 107:86728676

Stevens M, Troscianko J, Spottiswoode CN (2013) Repeated targeting of the same hosts by a brood parasite compromises host egg rejection. Nat Commun $4: 2475$

Stoddard MC, Stevens M (2011) Avian vision and the evolution of egg color mimicry in the common cuckoo. Evolution 65:2004-2013

Stoddard MC, Kilner RM, Town C (2014) Pattern recognition algorithm reveals how birds evolve individual egg pattern signatures. Nat Commun 5:4117

Stokke BG, Polačiková L, Dyrcz A, Hafstad I, Moksnes A, Røskaft E (2010) Responses of Reed Warblers Acrocephalus scirpaceus to non-mimetic eggs of 
different sizes in a nest parasitism experiment. Acta Ornithol 45:98-104

Tinbergen N, Broekhuysen GJ, Feekes F, Houghton JCW, Kruuk H, Szulc E (1962) Egg shell removal by the black-headed gull, Larus ridibundus L.: a behaviour component of camouflage. Behaviour 19:74-117

Vorobyev M, Osorio D (1998) Receptor noise as a determinant of colour thresholds. Proc R Soc Lond B 265:351-358

Yang C, Møller AP, Røskaft E, Moksnes A, Liang W, Stokke BG (2014) Reject the odd egg: egg recognition mechanisms in parrotbills. Behav Ecol 25:13201324

Zölei A, Hauber ME, Geltsch N, Moskát C (2012) Asymmetrical signal content of egg shape as predictor of egg rejection by great reed warblers, hosts of the common cuckoo. Behaviour 149:391-416 